\title{
Advancing the Use of Mobile Technologies in Clinical Trials: Recommendations from the Clinical Trials Transformation Initiative
}

\begin{abstract}
Philip Coran $^{a} \quad$ Jennifer C. Goldsack ${ }^{\text {b, u }}$ Cheryl A. Grandinetti ${ }^{c}$ Jessie P. Bakker ${ }^{d} \quad$ Marisa Bolognese ${ }^{e} \quad$ E. Ray Dorsey ${ }^{f}$ Kaveeta Vasisht ${ }^{c}$ Adam Amdurg Christopher Dell $^{\text {h }}$ Jonathan Helfgott ${ }^{i}$ Matthew Kirchoff ${ }^{j}$ Christopher J. Millerk Ashish Narayan' Dharmesh Patel ${ }^{m}$ Barry Peterson $^{n} \quad$ Ernesto Ramirez $^{\circ}$ Drew Schiller $^{p}$ Thomas Switzer ${ }^{q}$ Liz Wing $^{r}$ Annemarie Forrest ${ }^{b}$ Aiden Doherty ${ }^{s, t}$

a Medidata Solutions, New York, NY, USA; ${ }^{b}$ Clinical Trials Transformation Initiative, Durham, NC, USA; ' Center for Drug Evaluation and Research, US Food and Drug Administration, Silver Spring, MD, USA; ${ }^{d}$ Philips, Monroeville, PA, USA; ${ }^{\mathrm{T}}$ The Life Raft Group, Wayne, NJ, USA; ${ }^{f}$ Center for Health and Technology and Department of Neurology, University of Rochester Medical Center, Rochester, NY, USA; ${ }^{9}$ American Sleep Apnea Association, Washington, DC, USA; h Pfizer Inc., Collegeville, PA, USA; 'Johns Hopkins University, Baltimore, MD, USA; ${ }^{j}$ National Institute of Allergy and Infectious Diseases, National Institutes of Health, Bethesda, MD, USA; ${ }^{k}$ AstraZeneca, Gaithersburg, MD, USA; 'Icahn School of Medicine, Mount Sinai Health System, New York, NY, USA; ${ }^{\mathrm{m}}$ Center for Devices and Radiological Health, US Food and Drug Administration, Silver Spring, MD, USA; nIndependent Consultant, Fort Mill, SC, USA; ${ }^{\circ}$ Evidation Health, San Mateo, CA, USA; PValidic, Durham, NC, USA; ${ }^{9}$ Genentech Inc., South San Francisco, CA, USA; 'Duke Clinical

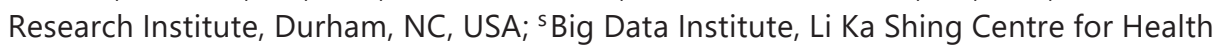
Information and Discovery, University of Oxford, Oxford, UK; ${ }^{\mathrm{t}}$ National Institute for Health Research, Oxford Biomedical Research Centre, Oxford University Hospitals National Health Service Foundation Trust, John Radcliffe Hospital, Oxford, UK; u Digital Medicine (DiMe) Society, New York, NY, USA
\end{abstract}

\section{Keywords}

Mobile technologies · Clinical trials · Wearable sensors $\cdot$ Regulatory approval

\section{Abstract}

Mobile technologies offer the potential to reduce the costs of conducting clinical trials by collecting high-quality information on health outcomes in real-world settings that are relevant to patients and clinicians. However, widespread use of mobile technologies in clinical trials has been impeded by their perceived challenges. To advance solutions to these challenges, the Clinical Trials Transformation Initiative (CTTI) has issued best practices and realistic approach- 
Coran et al.: Advancing the Use of Mobile Technologies in Clinical Trials:

Recommendations from the Clinical Trials Transformation Initiative

es that clinical trial sponsors can now use. These include CTTI recommendations on technology selection; data collection, analysis, and interpretation; data management; protocol design and execution; and US Food and Drug Administration submission and inspection. The scientific principles underpinning the clinical trials enterprise continue to apply to studies using mobile technologies. These recommendations provide a framework for including mobile technologies in clinical trials that can lead to more efficient assessment of new therapies for patients.

(C) 2019 The Author(s)

Published by S. Karger AG, Basel

\section{Introduction}

The ability of mobile technologies to passively and continuously collect health-relevant data holds much potential to improve the quality and efficiency of clinical research studies [1]. Mobile technologies are defined as wearables, ingestibles, implantables, portable sensors placed in the home, and smartphones with embedded sensors and applications optimized for electronic outcomes assessment. These technologies are designed to collect measures directly from an individual [2]. In the conduct of a US Food and Drug Administration (FDA)-regulated clinical trial, such technologies can potentially capture high-quality, continuous outcome measures from a study participant over the course of days, weeks, or months, while the participants go about their usual everyday activities. This could contribute to a more complete picture of a patient's experience than is typically obtained at discrete visits to a clinic or lab. This use of mobile technology to collect data, in addition to traditional efficacy data, can lead to improved assessment of response to treatment and thereby support more efficient development of new therapies for patients.

\section{The Potential of Mobile Technologies in Clinical Trials}

Duchenne's muscular dystrophy (DMD) is a rare genetic disorder characterized by progressive muscle degeneration and weakness that primarily affects boys and young men. Children are typically diagnosed at around 4 years of age, become unable to walk by age 12 , and have a life expectancy of 26 years. There is no curative treatment for DMD, and few disease-modifying therapies are available [3]. The North Star Ambulatory Assessment, a 17-item rating scale used to measure functional motor abilities, has increasingly replaced the 6-minute walk test as the standard assessment used in trials of medical products to treat DMD [4]. While the median age of loss of ambulation is approximately age 12, patients are living longer due to improved standard of care [5]. The addition of a mobile technology - such as an accelerometer worn on a limb, waist, or placed on the wheelchair, or a sensor embedded in a smartphone or gaming handset - could make possible the capture of real-time data about activity or movements that may not be measurable at the same frequency using other instruments. In addition, the ability to measure and transmit relevant data remotely has the potential to minimize the burden on participants and their caregivers in trials. This may facilitate the design of more efficient clinical trials that could enable greater patient participation and could encourage more sponsors to develop therapies for DMD [6-8].

Alzheimer's and other dementias currently affect 5 million Americans at a cost of USD 259 billion in $2017[9,10]$. By 2050, the prevalence is projected to increase to as many as 16 million people [8]. Failure rates in recent Alzheimer's disease clinical trials approached $100 \%$ [11]. A measure of cognition commonly used in Alzheimer's disease trials, developed in the 1980s, is the Alzheimer's Disease Assessment Scale-Cognitive (ADAS-Cog) [12]. However, 
this outcome measure may not be able to detect earlier stages of disease progression [13]. Given the nature of this condition, a continuous measurement of daily living activities in Alzheimer's patients could be more sensitive than relying on a single data point captured during clinic visits or even daily patient diary entries. A continuous measurement may provide early identification of disease progression when the therapies could be more effective [1416]. This could offer a way to assess effectiveness in drug development programs as well as improved efficiency of trial design [17].

\section{Addressing the Current Limited Use of Mobile Technologies in Clinical Trials}

Despite this apparent promise to better measure some important health outcomes, the use of mobile technologies remains underutilized in clinical trials $[18,19]$. Trial sponsors have noted important scientific and technical challenges that affect their decision-making around using a mobile technology for data capture. Concerns include how to choose the most appropriate mobile technology; how to collect, analyze, and interpret the data; how to ensure data authenticity, integrity, and confidentiality; how to design protocols that employ mobile technologies; and how to prepare supporting materials for regulatory submission [20].

To advance solutions to these challenges, the Clinical Trials Transformation Initiative (CTTI) issued recommendations and resources for appropriate use of mobile technologies in clinical trials for objective outcomes data capture (use of technology for recruitment, e-consent, ePROs, etc. were out of scope for the project). CTTI is a multi-stakeholder organization cofounded by Duke University and the FDA whose mission is to develop and drive adoption of practices that will increase the quality and efficiency of clinical trials. CTTI conducted 43 qualitative interviews [21] with clinical trial sponsors $(n=15)$, investigators $(n=8)$, device manufacturers $(n=10)$, data management experts $(n=2)$, biostatisticians $(n=4)$, and data security experts $(n=4)$. The Duke University Health System Institutional Review Board designated the interviews "exempt" from full board review. Data from those interviews, together with expertise of team members (regulatory, technical, clinical, operational, and patient experts) and input from expert meeting attendees [20], informed the consensus-based recommendations described herein.

\section{CTTI Recommendations on the Use of Mobile Technologies in Clinical Trials}

The scientific principles underpinning the clinical trials enterprise [22] continue to apply to trials using mobile technologies for data capture, as do data quality principles. Also, the active engagement of study participants remains crucial to the design and conduct of such trials. In this paper, we present recommendations in five key areas for the appropriate inclusion of mobile technologies in clinical trials: (1) technology selection, (2) data collection, analysis, and interpretation, (3) data management, (4) protocol design and execution, and (5) CTTI recommendations for FDA submission and inspection (Fig. 1). The complete set of recommendations and tools are available at the CTTI website [23].

\section{Mobile Technology Selection}

CTTI's framework for mobile technology selection [24] can assist sponsors in their decision-making process and is summarized in Figure 2. It is critical that sponsors first identify the aspect or experience the assessment is intended to measure (i.e., an individual's 
... 미미리

Diømarkers

\begin{tabular}{l|l}
\hline Digit Biomark 2019;3:145-154 \\
\hline DOI: 10.1159/000503957 & $\begin{array}{l}\text { @ } 2019 \text { The Author(s). Published by S. Karger AG, Basel } \\
\text { www.karger.com/dib }\end{array}$ \\
\hline
\end{tabular}

Coran et al.: Advancing the Use of Mobile Technologies in Clinical Trials:

Recommendations from the Clinical Trials Transformation Initiative

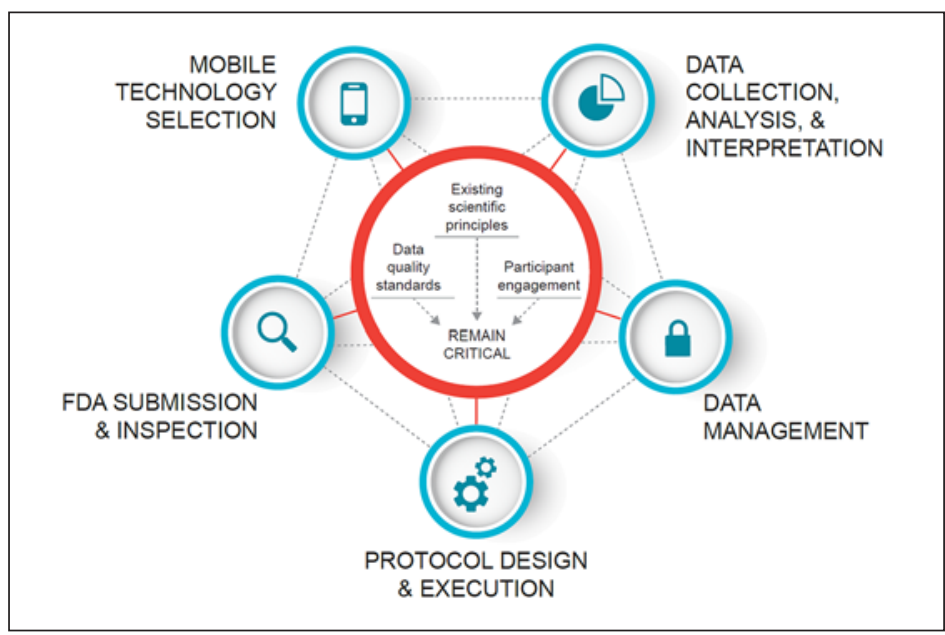

Fig. 1. Mobile technology considerations.
Fig. 2. Framework for mobile technology selection.

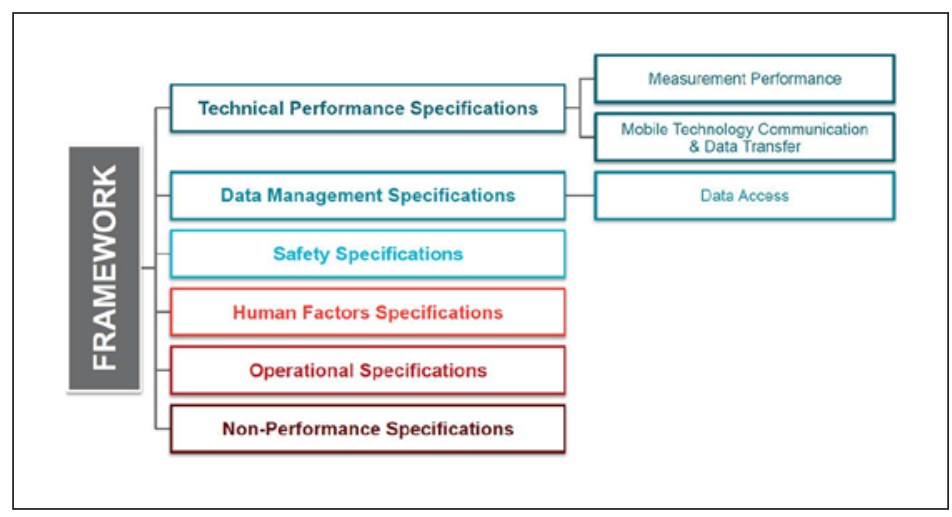

clinical, biological, physical, or functional state). Only then should sponsors consider the technical performance specifications and functional characteristics needed to measure the outcome. The processes of verification (the mobile technology reliably measures what it claims to measure) and validation (the processed data being assessed are suitable for its intended use and patient population in a trial) ensure that the mobile technology is scientifically justifiable for use in a certain population and setting.

Sponsors should consider conducting feasibility studies prior to launching the trial. These studies may assess the suitability of the mobile technology and identify any unanticipated issues when used in context, such as tolerability, acceptability, and usability in the trial population. Feasibility studies will also provide an opportunity for the study team to become familiar with the technical aspects of the devices (e.g., battery life) and the data outputs as well as evaluate the quality and completeness of data collected. CTTI recently conducted a systematic review (https://www.nature.com/articles/s41746-019-0125-x) and compiled a database (https://feasibility-studies.ctti-clinicaltrials.org) summarizing pilot studies addressing mobile technology sensor performance, algorithm development, software performance, and/or operational feasibility, in order to provide a resource for guiding decisions about which technology is most suitable for a particular trial.

Finally, if mobile technologies will be used to capture a clinical endpoint for a regulatory decision, such as labeling, it is important to engage with regulatory authorities early to discuss the appropriateness of the endpoint being captured and the specific validation process needed for use of the mobile technology to capture that endpoint. 
Coran et al:: Advancing the Use of Mobile Technologies in Clinical Trials:

Recommendations from the Clinical Trials Transformation Initiative

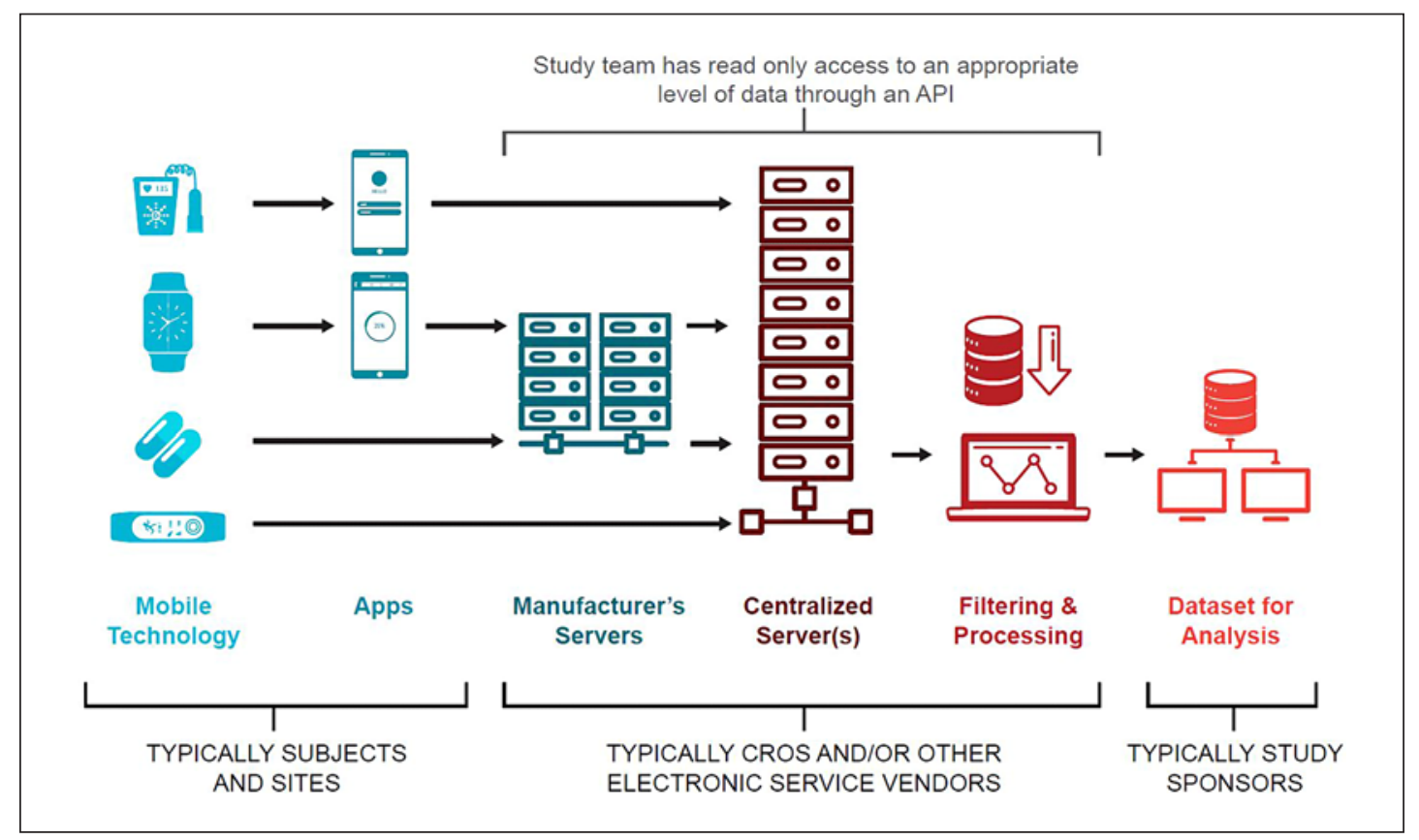

Fig. 3. Data flow diagram.

\section{Data Collection, Analysis, and Interpretation}

The volume and structure of data collected continuously and remotely is much greater than what is typically collected in a traditional trial. Therefore, it is essential that sponsors engage the skills of biostatisticians and data scientists in decisions regarding protocol design, data collection, analysis, and interpretation. Only high-quality, clinically meaningful data that are analyzed appropriately can inform robust conclusions. Appropriate data quality optimization approaches will help minimize data variability, reduce missing data captured from the mobile technology, and ensure that data are collected from the intended participant. CTTI's recommended strategies for optimizing data quality provide best practices for sponsors [25].

\section{Data Management}

CTTI's resources illustrate the complex flow of data in trials using mobile technologies (Fig. 3) and offer strategies for promoting and protecting data integrity across the entire data life cycle [26]. Data must be secured both on the mobile technology itself and during the transfer of data. Sponsors are ultimately responsible for data management, but many data management processes may be carried out by, or in partnership with, contract research organizations or other third parties, such as information technology service providers (e.g., Platform as a Service, Software as a Service, and Infrastructure as a Service) and mobile technology manufacturers. Thus, it is critical to have a full understanding of the data flow and know who is responsible for the data and accountable for data integrity at each step during the data life cycle.

In each step of the flow of data - from the point of collection by a mobile technology through to the production of datasets for analysis - sponsors will need various methods to ensure data integrity (e.g., access controls, implementation, maintenance and retention of 
Coran et al.: Advancing the Use of Mobile Technologies in Clinical Trials: support or contextualizing information, understand the data?

Example: An EKG wave vs. step count

Is sharing and/or displaying data in real time part of standard care?

Example: Use of a CGM in T1D

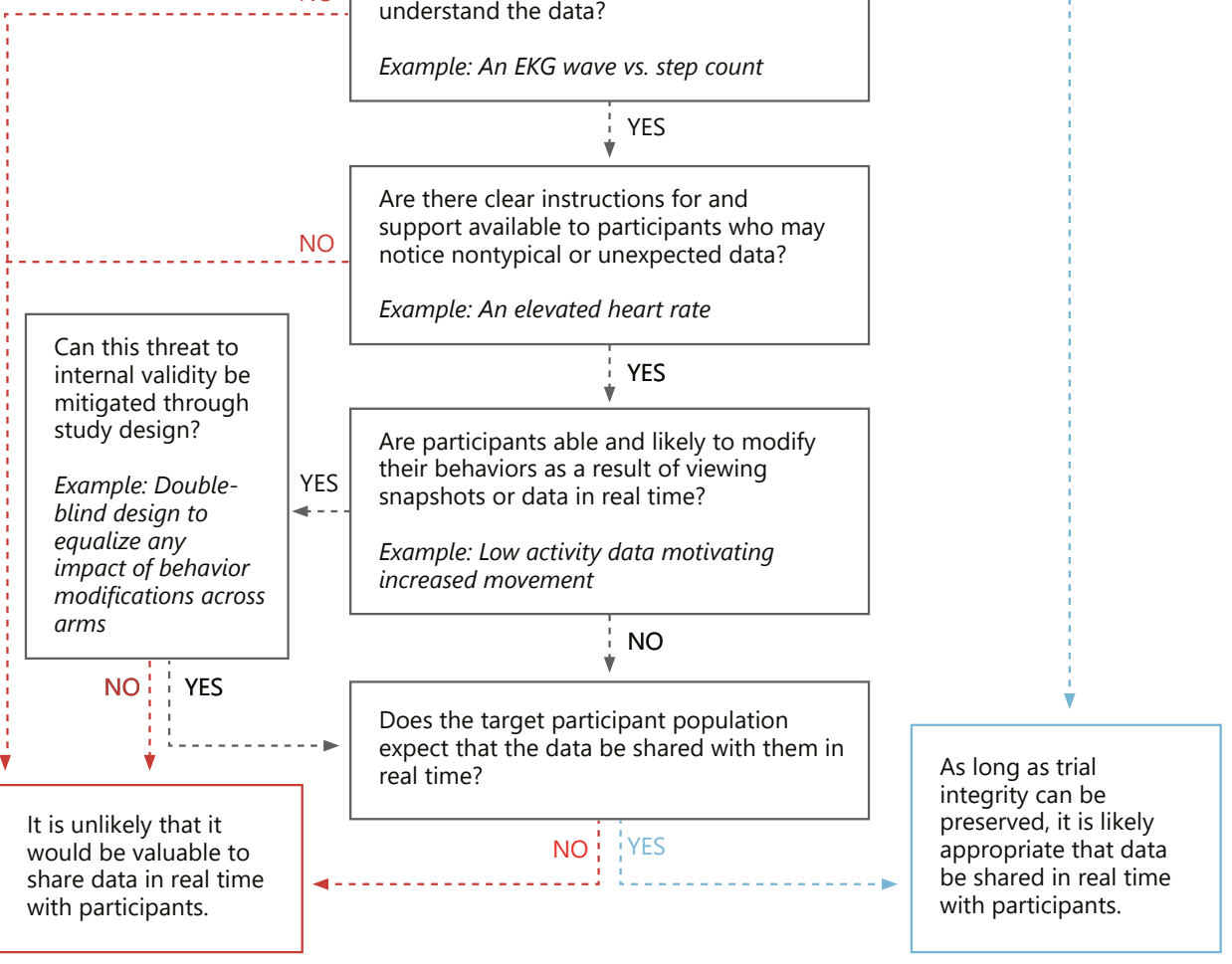

Fig. 4. Decision support tool: real-time data sharing with study participants.

audit trails, end-to-end data encryption, user training). It is important to note that there is not a "one size fits all" approach to data security, and data security solutions should be developed with the entire data flow and management infrastructure in mind. CTTI has recommended practical approaches for sponsors to secure data generated by mobile technologies [27].

\section{Protocol Design and Execution}

When incorporating mobile technologies for data capture in trials, sponsors will make decisions on whether or how to share real-time data with study participants. It is essential that any data sharing preserves trial integrity and internal validity and does not affect the safety, quality, and feasibility of the study. CTTI has developed a decision support tool that sponsors can adapt to their specific trial needs for data sharing with participants (Fig. 4).

Using mobile technologies for remote data capture offers the possibility of collecting timely and complete safety information about an investigational medical product. In 
Coran et al.: Advancing the Use of Mobile Technologies in Clinical Trials:

Recommendations from the Clinical Trials Transformation Initiative

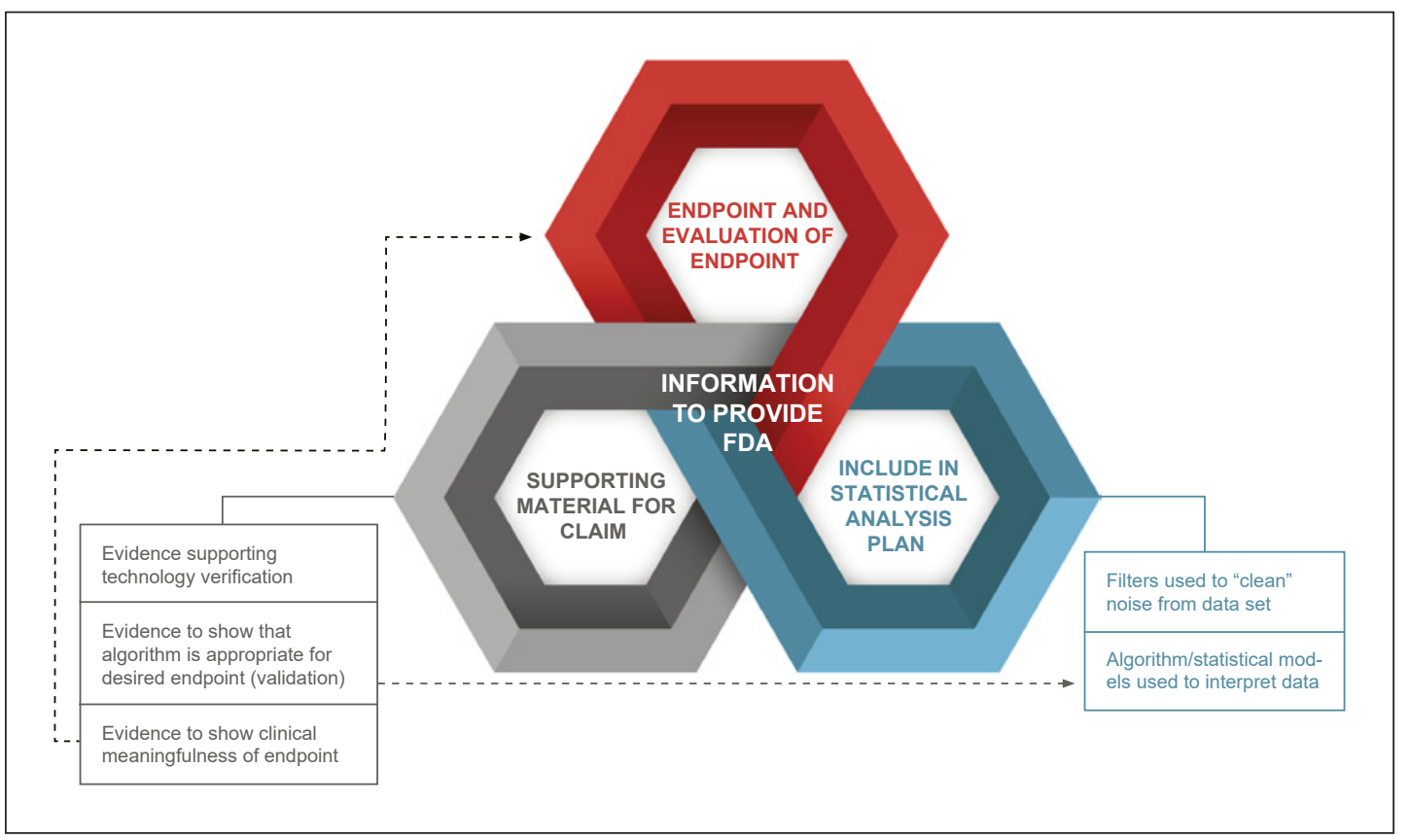

Fig. 5. CTTI recommendations for data processes and information to provide to FDA.

contrast to traditional trials, the use of mobile technologies raises two new potential issues related to safety signals that sponsors should consider. The first is around safety signals not previously observed using traditional protocol design and monitoring. The second is on data collected and observed by the study participant, in the absence of context provided by clinicians, which may lead to difficulty distinguishing between normal data and a possible adverse event. CTTI has developed a framework of options and approaches to assist sponsors seeking to optimize their approach for managing atypical data, including those data captured outside of the intended use of the mobile technology [28]. Study participants should be well informed regarding safety monitoring and its enablement through mobile technologies.

\section{CTTI Recommendations for FDA Submission and Inspection}

Figure 5 summarizes data and the data processes and information that should generally be included in a marketing submission. To ensure success in a premarket submission for a new drug or medical device that includes studies using mobile sensor technology for data capture, it will be important for sponsors to engage with the FDA early in the process of trial design. The most appropriate strategy for collecting and sharing data with the FDA will likely be trial-specific and require an open dialogue prior to and during trial conduct.

Source data should generally be the primary data resource provided to the FDA. This data should be the first level of data that is both clinically relevant to the trial and interpretable, and this should be prespecified in the data collection plan. Metadata and audit trails should be maintained and retained. 


\section{Summary}

There is reason to be optimistic about the positive transformation that mobile technologies can bring to the clinical trials enterprise. Mobile technologies are in a unique position to offer high-quality, real-world information and new insights on outcomes that are relevant to patients and clinicians, in addition to reducing the costs of conducting clinical research [29].

While incorporating mobile technologies for data capture will not be the solution to every challenge faced in the conduct of clinical trials, where benefit exists, CTTI's recommendations will support more successful implementation of mobile clinical trials. The scientific principles underpinning the clinical trials enterprise continue to apply to trials using mobile technologies. For clinical trial sponsors interested in adopting and optimizing the use of mobile technologies for data capture, the full set of recommendations, tools, and resources can be downloaded from CTTI [23].

\section{Acknowledgements}

The authors would like to thank Pat Furlong, President and CEO of Parent Project Muscular Dystrophy (PPMD), and Abby Bronson, MBA, Senior Vice President, Research Strategy at PPMD, for their review and feedback on the description of Duchenne muscular dystrophy. The authors also would like to thank Amy Corneli and Brian Perry, Duke University, Department of Population Health Sciences, for their leadership of data collection activities informing development of the recommendations and resources.

\section{Statement of Ethics}

The authors have no ethical conflicts to disclose.

\section{Disclosure Statement}

J.C.G., C.A.G., J.P.B., M.B., K.V., A.A., C.D., J.H., M.K., A.N., D.P., T.S., L.W., and A.D. have no conflicts of interest to declare. P.C. is an employee of Medidata Solutions with ownership interests. E.R.D. has received honoraria for speaking at American Academy of Neurology courses, American Neurological Association, and University of Michigan; received compensation for consulting services from 23andMe, Abbott, Abbvie, American Well, Biogen, Clintrex, DeciBio, Denali Therapeutics, GlaxoSmithKline, Grand Rounds, Karger, Lundbeck, MC10, MedAvante, Medical-legal services, Mednick Associates, National Institute of Neurological Disorders and Stroke, Olson Research Group, Optio, Prilenia, Putnam Associates, Roche, Sanofi, Shire, Sunovion Pharma, Teva, UCB, and Voyager Therapeutics; research support from Abbvie, Acadia Pharmaceuticals, AMC Health, Biosensics, Burroughs Wellcome Fund, Davis Phinney Foundation, Duke University, Food and Drug Administration, GlaxoSmithKline, Greater Rochester Health Foundation, Huntington Study Group, Michael J. Fox Foundation, National Institutes of Health/National Institute of Neurological Disorders and Stroke, National Science Foundation, Nuredis Pharmaceuticals, Patient-Centered Outcomes Research Institute, Pfizer, Prana Biotechnology, Raptor Pharmaceuticals, Roche, Safra Foundation, Teva Pharmaceuticals, University of California Irvine; editorial services for Karger Publications; and ownership interests with Blackfynn (data integration company) and Grand Rounds (second opinion service). C.J.M. is an employee of AstraZeneca and a shareholder of AstraZeneca, 
Coran et al.: Advancing the Use of Mobile Technologies in Clinical Trials:

Recommendations from the Clinical Trials Transformation Initiative

Abbott, Abbvie, and J\&J. B.P. was at Philips Respironics from 2011 to 2017. D.S. has ownership interests in Validic. E.R. is an employee of Evidation Health. J.P.B. has been a full-time employee at Philips since 2017.

\section{Funding Sources}

Funding for the manuscript was made possible, in part, by the Food and Drug Administration through grant R18FD005292. Views expressed in written materials do not necessarily reflect the official policies of the Department of Health and Human Services, nor does any mention of trade names, commercial practices, or organization imply endorsement by the United States Government. Partial funding was also provided by pooled membership fees and in-kind contributions from CTTI's member organizations. A.D. is supported by the National Institute for Health Research (NIHR) Oxford Biomedical Research Centre (BRC) and Health Data Research UK.

\section{Author Contributions}

P.C., J.C.G., C.A.G., J.P.B., M.B., E.R.D., K.V., A.A., C.D., J.H., M.K., C.J.M., A.N., D.P., B.P., E.R., D.S., T.S., and A.D. developed the conceptual framework, recommendations, and tools. J.C.G., A.F., and L.W. drafted the manuscript with input from all authors. All authors provided critical review and approval of the submitted version.

\section{References}

1 Perry B, Herrington W, Goldsack JC, Grandinetti CA, Vasisht KP, Landray MJ, et al. Use of Mobile Devices to Measure Outcomes in Clinical Research, 2010-2016: A Systematic Literature Review. Digit Biomark. 2018 Jan; 2(1):11-30.

2 Doherty A, Jackson D, Hammerla N, Plötz T, Olivier P, Granat MH, et al. Large Scale Population Assessment of Physical Activity Using Wrist Worn Accelerometers: The UK Biobank Study. PLoS One. 2017 Feb; 12(2):e0169649.

3 Moxley RT 3rd, Pandya S, Ciafaloni E, Fox DJ, Campbell K. Change in natural history of Duchenne muscular dystrophy with long-term corticosteroid treatment: implications for management. J Child Neurol. 2010 Sep; 25(9):1116-29.

4 Ricotti V, Ridout DA, Pane M, Main M, Mayhew A, Mercuri E, et al.; UK NorthStar Clinical Network. The NorthStar Ambulatory Assessment in Duchenne muscular dystrophy: considerations for the design of clinical trials. J Neurol Neurosurg Psychiatry. 2016 Feb;87(2):149-55.

5 Ryder S, Leadley RM, Armstrong N, Westwood M, de Kock S, Butt T, et al. The burden, epidemiology, costs and treatment for Duchenne muscular dystrophy: an evidence review. Orphanet J Rare Dis. 2017 Apr;12(1):79.

6 Dolgin E. Industry embraces virtual trial platforms. Nat Rev Drug Discov. 2018 Apr;17(5):305-6.

7 Dorsey ER, Venuto C, Venkataraman V, Harris DA, Kieburtz K. Novel methods and technologies for 21st-century clinical trials: a review. JAMA Neurol. 2015 May;72(5):582-8.

8 Smalley E. Clinical trials go virtual, big pharma dives in. Nat Biotechnol. 2018 Jul;36(7):561-2.

9 Feigin VL, Abajobir AA, Abate KH, Abd-Allah F, Abdulle AM, Abera SF, et al.; GBD 2015 Neurological Disorders Collaborator Group. Global, regional, and national burden of neurological disorders during 1990-2015: a systematic analysis for the Global Burden of Disease Study 2015. Lancet Neurol. 2017 Nov;16(11):877-97.

10 Alzheimer's Association. 2017 Alzheimer's Disease Facts and Figures. March 2017. Available at: https://www. alz.org/email/alz-media-insider/downloads/2017-FF-Factsheet.pdf. Accessed October 29, 2018.

11 Rosenblatt M, Termeer H. Reframing the Conversation on Drug Pricing. November 2, 2017. NEJM Catalyst. Available at: https://catalyst.nejm.org/reframing-conversation-drug-pricing/. Accessed November 8, 2018.

12 Rosen WG, Mohs RC, Davis KL. A new rating scale for Alzheimer's disease. Am J Psychiatry. 1984 Nov; 141(11): 1356-64.

13 Kueper JK, Speechley M, Montero-Odasso M. The Alzheimer's Disease Assessment Scale-Cognitive Subscale (ADAS-Cog): Modifications and Responsiveness in Pre-Dementia Populations. A Narrative Review. J Alzheimers Dis. 2018;63(2):423-44. 
Coran et al.: Advancing the Use of Mobile Technologies in Clinical Trials:

Recommendations from the Clinical Trials Transformation Initiative

14 Bateman DR, Srinivas B, Emmett TW, Schleyer TK, Holden RJ, Hendrie HC, et al. Categorizing Health Outcomes and Efficacy of mHealth Apps for Persons With Cognitive Impairment: A Systematic Review. J Med Internet Res. 2017 Aug;19(8):e301.

15 Leurent C, Ehlers MD. Digital technologies for cognitive assessment to accelerate drug development in Alzheimer's disease. Clin Pharmacol Ther. 2015 Nov;98(5):475-6.

16 Dodge HH, Zhu J, Mattek NC, Austin D, Kornfeld J, Kaye JA. Use of High-Frequency In-Home Monitoring Data May Reduce Sample Sizes Needed in Clinical Trials. PLoS One. 2015 Sep;10(9):e0138095.

17 Gold M, Amatniek J, Carrillo MC, Cedarbaum JM, Hendrix JA, Miller BB, et al. Digital technologies as biomarkers, clinical outcomes assessment, and recruitment tools in Alzheimer's disease clinical trials. Alzheimers Dement (N Y). 2018 May;4:234-42.

18 Steinhubl SR, Topol EJ. Digital medicine, on its way to being just plain medicine. npj Digital Medicine 2018;1: 20175.

19 Topol EJ. The Patient Will See You Now: The Future of Medicine Is in Your Hands. Basic Books; 2016.

20 Clinical Trials Transformation Initiative. Mobile Clinical Trials (MCT). Mobile Technologies. Scientific and Technological Issues Surrounding the Use of Mobile Devices in Clinical Trials [meeting materials]. Available at: https://www.ctti-clinicaltrials.org/briefing-room/meetings/scientific-and-technological-issuessurrounding-use-mobile-devices-clinical. Accessed March 11, 2019.

21 Clinical Trials Transformation Initiative. Mobile Clinical Trials (MCT). Mobile Technologies. Presentation of Evidence on Data Challenges [slide set]. June 15, 2017. Available at: https://www.ctti-clinicaltrials.org/ sites/www.ctti-clinicaltrials.org/files/1.1_panel_presentation-of-evidendce_mct-mobiledevices.pdf. accessed March 11, 2019.

22 Peto R, Pike MC, Armitage P, Breslow NE, Cox DR, Howard SV, et al. Design and analysis of randomized clinical trials requiring prolonged observation of each patient. I. Introduction and design. Br J Cancer. 1976 Dec;34(6): 585-612.

23 Clinical Trials Transformation Initiative. Mobile Clinical Trials (MCT): Mobile Technologies. Available at: https://www.ctti-clinicaltrials.org/projects/mobile-technologies. Accessed October 29, 2018.

24 Clinical Trials Transformation Initiative. Mobile Clinical Trials (MCT): Mobile Technologies. Framework of Specifications to Consider During Mobile Technology Selection. Available at https://www.ctti-clinicaltrials. org/projects/mobile-technologies. Accessed November 8, 2018.

25 Clinical Trials Transformation Initiative. Mobile Clinical Trials (MCT): Mobile Technologies. Strategies for Optimizing Data Quality. Available at: https://www.ctti-clinicaltrials.org/projects/mobile-technologies. Accessed November 8, 2018.

26 Clinical Trials Transformation Initiative. Mobile Clinical Trials (MCT). Mobile Technologies. Strategies for Promoting and Protecting Data Integrity. Available at: https://www.ctti-clinicaltrials.org/projects/mobiletechnologies. Accessed November 8, 2018.

27 Clinical Trials Transformation Initiative. Mobile Clinical Trials (MCT). Mobile Technologies. Approaches to Securing Data Generated by Mobile Technologies. Available at: https://www.ctti-clinicaltrials.org/projects/ mobile-technologies. Accessed November 8, 2018.

28 Clinical Trials Transformation Initiative. Mobile Clinical Trials (MCT). Mobile Technologies. Framework of Approaches for Safety Monitoring and Managing Safety Signals. Available at: https://www.ctti-clinicaltrials. org/projects/mobile-technologies. Accessed November 8, 2018.

29 Steinhubl SR, McGovern P, Dylan J, Topol EJ. The digitised clinical trial. Lancet. 2017 Nov;390(10108):2135. 